\title{
Beam stabilization in the two-dimensional nonlinear Schrodinger equation with an attractive potential by beam splitting and radiation
}

leMesurier, B.J.; Christiansen, Peter Leth; Gaididei, Yuri Borisovich; Juul Rasmussen, Jens

Published in:

Physical Review E

Link to article, DOI:

10.1103/PhysRevE.70.046614

Publication date:

2004

Document Version

Publisher's PDF, also known as Version of record

Link back to DTU Orbit

Citation (APA):

leMesurier, B. J., Christiansen, P. L., Gaididei, Y. B., \& Juul Rasmussen, J. (2004). Beam stabilization in the two-dimensional nonlinear Schrodinger equation with an attractive potential by beam splitting and radiation. Physical Review E, 70(4), 046614 (7 pages). https://doi.org/10.1103/PhysRevE.70.046614

\section{General rights}

Copyright and moral rights for the publications made accessible in the public portal are retained by the authors and/or other copyright owners and it is a condition of accessing publications that users recognise and abide by the legal requirements associated with these rights.

- Users may download and print one copy of any publication from the public portal for the purpose of private study or research.

- You may not further distribute the material or use it for any profit-making activity or commercial gain

- You may freely distribute the URL identifying the publication in the public portal 


\title{
Beam stabilization in the two-dimensional nonlinear Schrödinger equation with an attractive potential by beam splitting and radiation
}

\author{
Brenton John leMesurier* \\ Department of Mathematics, College of Charleston, Charleston, South Carolina 29424, USA \\ Peter Leth Christiansen ${ }^{\dagger}$ \\ Informatics and Mathematical Modelling and Department of Physics, Technical University of Denmark, DK-2800 Kgs Lyngby, Denmark \\ Yuri B. Gaididei \\ Bogolyubov Institute for Theoretical Physics, 252143 Kiev, Ukraine \\ Jens Juul Rasmussen \\ Department of Optics and Plasma Research, OPL-128, Risф National Laboratory, P.O. Box 49, DK-4000 Roskilde, Denmark \\ (Received 14 August 2003; revised manuscript received 14 June 2004; published 28 October 2004)
}

\begin{abstract}
The effect of attractive linear potentials on self-focusing in-waves modeled by a nonlinear Schrödinger equation is considered. It is shown that the attractive potential can prevent both singular collapse and dispersion that are generic in the cubic Schrödinger equation in the critical dimension 2 and can lead to a stable oscillating beam. This is observed to involve a splitting of the beam into an inner part that is oscillatory and of subcritical power and an outer dispersing part. An analysis is given in terms of the rate competition between the linear and nonlinear focusing effects, radiation losses, and known stable periodic behavior of certain solutions in the presence of attractive potentials.
\end{abstract}

DOI: 10.1103/PhysRevE.70.046614

PACS number(s): 42.65.Jx

\section{INTRODUCTION}

Variants of the nonlinear Schrödinger equation (NLSE) with attractive potentials arise in many models of weakly nonlinear dispersive waves and the nonlinear phenomenon of self-focusing or wave collapse. The simplest NLSE with only a cubic nonlinearity has unusual behavior such as the formation of self-focusing singularities in finite time when there are two or more dimensions transverse to the propagation variable. The problem is most acute for critical transverse dimension $D=2$, which is fraught with instabilities: small perturbations in initial data or various small additional terms modeling physical features ignored in the basic cubic Schrödinger equation (CSE) can change solutions between singular collapse and dispersion.

Most such perturbations of the equation are higher-order nonlinear effects, but here we consider the effects of an attractive potential, giving what will be called the nonlinear Schrödinger equation with potential (NLSP):

$$
i \frac{\partial \psi}{\partial t}+\nabla^{2} \psi+|\psi|^{2} \psi-U(\mathbf{x}) \psi=0
$$

We shall concentrate on the two-dimensional (2D) case with $\psi=\psi(t, x, y), \nabla^{2}=\partial_{x}^{2}+\partial_{y}^{2}$. This arises in models of $\mathrm{cw}$ laser propagation in waveguides, molecular excitations in a lattice near an inhomogeneity [1], the Gross-Pitaevskii model [2]

\footnotetext{
*Electronic address: lemesurierb@cofc.edu

†Electronic address: plc@imm.dtu.dk

"Electronic address: jens.juul.rasmussen@ risoe.dk
}

for attractive quasi-2D Bose-Einstein condensates (BEC), and magnetic nanostructures [3]. The standard GrossPitaevskii equation for BEC's in a confining trap has a quadratic potential and, usually, also linear and nonlinear dissipation terms, but the latter terms are unimportant to the current study. Note that, in some of the applications, $t$ represents the propagation distance rather than time. The cubic nonlinearity represents the intensity-dependent refractive index in the optical case, exciton-phonon interaction in molecular excitations, and interaction between the Bose particles in BEC: the last is repulsive in most cases, but attractive for ${ }^{7} \mathrm{Li}$ atoms for example. The potential $U(\mathbf{x})$ represents a spatially dependent refractive index for laser propagation, spatial inhomogeneity for molecular excitations, and the confining field or "trap" used in forming a BEC.

In situations where the initial beam is wide relative to the potential, one convenient model for the potential is a Gaussian

$$
U(r)=-h_{p} e^{-r^{2} /\left(2 w_{p}^{2}\right)}
$$

of depth $h_{p}$, and width $w_{p}$. This is plausible for a graded index fiber laser waveguide and for molecular excitations, where the potential represents the effect of a small impurity in the molecular lattice. No corresponding results from physical experiments are known to the authors, but the results here can give indications of experimental parameters to try, for example, indicating that the potentials should be somewhat narrower than the initial self-induced potential $-\left|\psi^{2}\right|$.

Recent numerical observations [4-7] show that such an attractive Gaussian potential can have the counterintuitive 


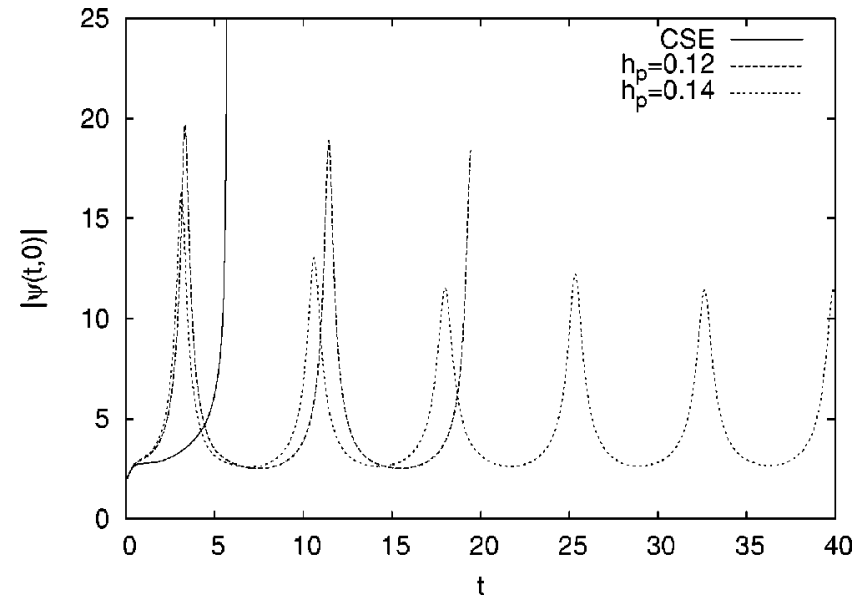

FIG. $1 . h=1.95, w_{p}=0.5$ : oscillations in the place of collapse.

effect of inhibiting or limiting collapse, while also preventing dispersion, leading instead to oscillatory beams that avoid both the extremes of singular collapse and dispersion that are the only generic outcomes in the focusing 2D CSE. This happens for various potentials narrower than the initial beam but sufficiently deep, with an initial focusing followed by a train of focusing and defocusing oscillations. For example, Fig. 1 shows the amplitude evolution for a case in which the CSE develops a singularity at $t \approx 5.73$, and Table I gives the shallowest potentials needed to inhibit singularity formation for various combinations of potential width and initial data. In that table, $T_{C S E}$ is the observed time of singularity formation for the CSE with the same initial data and $T_{t p}$ is the time of the first turning point of the intensity, when the beam first starts spreading again.

All simulations here are performed with Gaussian initial data centered at the origin, normalized to height $h$ and width 1: $\psi(0, r)=h e^{-r^{2} / 2}, r=|\mathbf{x}|$, and one can achieve this collapse inhibition with $h$ up to 2.5 , whereas without the potential, singularity formation is observed for all $h>h_{c} \approx 1.93$. The numerical methods used are described in $[4,8]$.

In the present paper we present additional numerical studies of the beam dynamics in the presence of an external potential. Particularly, we have invoked more detailed diagnostics to reveal the structure of the solution and the associated radiation. The main point of this paper is to present a detailed analysis explaining the beam dynamics in the potential. The analysis is based on a scaling argument and a collective coordinate calculation. For this, some background is needed; sources for results stated without citation are the articles of Rasmussen and Rypdal $[9,10]$ and the book of
Sulem and Sulem [11], as well as $[1,12,13]$ for more recent results involving also the influence of quadratic potentials.

Note that since our initial data are of the form $h e^{-r^{2} / 2}$, the conserved quantities of the CSE are the "power" $\mathcal{N}$ $=\int|\psi|^{2} d \mathbf{x}=\pi h^{2}$ and the Hamiltonian "energy" $\mathcal{H}=\mathcal{H}_{0}$ $:=\int\left[|\nabla \psi|^{2}-\frac{1}{2}|\psi|^{4}\right] d \mathbf{x}=\mathcal{N}(1-\mathcal{N} / 4 \pi)$. Thus the sufficient condition for global existence holds for $h<h_{c r}:=\sqrt{\mathcal{N}_{c r} / \pi}$ $\approx 1.927$, and singular collapse is guaranteed for $\mathcal{H}<0$ for $\mathcal{N}>4 \pi$, or $h>2$. Here $\mathcal{N}_{c r}$ is the critical power, which is given as the norm of the stationary ground state solution of the CSE. Also, the variance $V=\int r^{2}|\psi|^{2} d \mathbf{x}$ can be related to the beam width $\bar{r}$ defined through $V=\mathcal{N} \bar{r}^{2}$.

When a potential is added, exact results on singular collapse in (NLSP) are only known for the case of an attractive quadratic potential, which, however, is also a relevant approximation for a beam concentrated near the bottom of an attractive radially symmetric potential. The Gaussian potential (2) can be shifted by a harmless constant and then approximated near the origin by $U(r)=\Omega_{0}^{2} r^{2} / 4, \Omega_{0}^{2}=2 h_{p} / w_{p}^{2}$, so we will discuss quadratic potentials in this form. The power $\mathcal{N}$ is conserved with the same form as before, the conserved energy takes the form $\mathcal{H}=\mathcal{H}_{0}+\left(\Omega_{0} / 2\right)^{2} V$, and the variance satisfies

$$
V(t)=\left(V(0)-\frac{2 \mathcal{H}}{\Omega_{0}^{2}}\right) \cos 2 \Omega_{0} t+\frac{2 \mathcal{H}}{\Omega_{0}^{2}},
$$

as shown by several authors [12-15], so collapse is guaranteed if $\mathcal{H}_{0}(0)=\mathcal{H}-\Omega_{0}^{2} V(0) / 4 \leqslant 0$. The only change from the CSE is extending the singularity formation sufficient condition $\mathcal{H}_{0}(0)<0$ on the initial data to also include the case of equality. Note that if no singularity forms, the variance is bounded and in fact periodic.

Another kind of periodic solutions has been established by Rose and Weinstein [16]: orbitally stable steady-state solutions $e^{i \lambda t} \psi(r)$ with $\mathcal{N}<\mathcal{N}_{c}$. These always exist in the present situation, since that paper guarantees existence if the corresponding linear Schrödinger equation has a bound state, and in two dimensions this is true for any weak attractive potential, such as the attractive Gaussians used here.

\section{COLLAPSE INHIBITION: COMPETING COLLAPSE LENGTH SCALES AND RADIATION FROM SOLITON-LIKE SOLUTIONS}

\section{A. Argument for inhibition with approximate threshold, based on competing length scales}

We first discuss the inhibition of the collapse by a narrow potential using a simple scaling argument. The basic idea is

TABLE I. Minimum potential depth $h_{p}^{*}$ for collapse inhibition for various initial data and potential widths, with $T_{C S E}$ the observed collapse time in the absence of potential, and $T_{t p}$ the first turning time of intensity with potential.

\begin{tabular}{|c|c|c|c|c|c|c|c|c|c|c|c|}
\hline$h$ & 1.95 & 2 & & & & 2.1 & & & 2.2 & & 2.5 \\
\hline$T_{C S E}$ & 5.732 & 1.648 & & & & 0.9173 & & & 0.6775 & & 0.4063 \\
\hline$w_{p}$ & 0.4 & 0.35 & 0.2 & 0.1 & 0.01 & 0.3 & 0.05 & 0.02 & 0.2 & 0.05 & 0.15 \\
\hline$h_{p}^{*}$ & 0.15 & 1.3 & 1.75 & 4.5 & 230 & 3.46 & 30 & 100 & 7 & 70 & 20 \\
\hline$T_{t p}$ & 3.077 & 0.953 & 0.999 & 0.971 & 1.077 & 0.550 & 0.553 & 0.654 & 0.403 & 0.312 & 0.221 \\
\hline
\end{tabular}


comparing the numerically observed time scale $T_{C S E}$ for the self-focusing of the beam in the unmodified CSE with estimates of the time scale $T_{p o t}$ of the linear focusing and possible beam width oscillations in the case with potential, through approximations by the quadratic potential case.

When the potential is narrower than the initial beam $\left.\left(w_{p}<1\right)\right)$ and $T_{p o t}<T_{C S E}$, the beam may be split into inner and outer parts before a singularity can develop, with the faster focusing within the central part of the potential drawing part of the beam power and leaving behind a part focusing mostly under the slower nonlinear effect. If the inner part separates substantially from the outer and the power in the inner part is less than the critical power $\mathcal{N}_{c r}$ needed for formation of a focusing singularity, the initially fast focusing of the inner part driven by the potential would not be capable of continuing to singularity formation: instead its width could be expected to follow roughly the sinusoidal form in Eq. (3). Also the outer part can be expected to radiate outwards, particularly if it also has a subcritical share of total beam power, leaving an inner part of the beam of permanently subcritical power isolated in the central well of the potential, incapable of singular collapse and constrained from dispersion by the potential. This could leading to continued oscillation of this part's width and intensity, similar to that known to occur for a subcritical beam in a quadratic potential.

The singularity time $T_{C S E}$ is determined from simulations. Two approximate time scales for the focusing within the central part of the potential are suggested heuristically by comparing to quadratic potentials.

For the same initial data with the quadratic potential fitted at the origin to the Gaussian potential, the sinusoidal variance evolution (3) gives a first minimum of variance at $T_{p o t}:=\pi w_{p} / \sqrt{8 h_{p}}$, so by this time either a singularity forms or the beam starts spreading again. Note that this time depends only on the linear potential, once the nonlinearity has initiated focusing of the initial plane wave, and so for suitable potentials it can be far smaller than $T_{C S E}$.

However, the quadratic potential approximation only applies when a significant part of the beam's power lies within the concave part of the Gaussian potential, so this estimate should only apply when $w_{p}$ is not much smaller than 1 . Since this time scale relates to the width scale $w_{p}$ on which focusing occurs, a heuristic modification is to replace this by the actual, larger width scale $w$ of the beam, giving a slower time scale $T_{p o t}^{\prime}:=\pi w / \sqrt{8 h_{p}}$.

Either form of the time scale estimate suggests that for given initial data and potential width, collapse is likely to be inhibited for potential depth $h_{p}$ greater than some threshold $h_{p}^{*}$. This threshold behavior is observed in many cases, and Table II lists approximate values for various combination of beam height $h$ and potential width $w_{p}$, with the three estimates of potential focusing time scale and the observed time $T_{t p}$ of the first turning point (maximum intensity).

The second estimate $T_{p o t}^{\prime}$ is quite close to the observed turning point time, even when the potential width is significantly narrower than the initial beam. Also, the minimum potential depth needed for collapse inhibition is approximated by
TABLE II. Shallowest potentials for collapse inhibition for various initial data, with the observed time of the first turning point of intensity $T_{t p}$ and several versions of an estimate $T_{p o t}$ for this.

\begin{tabular}{cllllc}
\hline \hline$h$ & 1.95 & 2 & 2.1 & 2.2 & 2.5 \\
$w_{p}$ & 0.4 & 0.35 & 0.3 & 0.2 & 0.15 \\
$h_{p}^{*}$ & 0.15 & 1.3 & 3.46 & 7 & 20 \\
$T_{C S E}$ & 5.732 & 1.648 & 0.917 & 0.678 & 0.406 \\
$T_{t p}$ & 3.077 & 0.953 & 0.550 & 0.403 & 0.221 \\
$T_{p o t}$ & 1.147 & 0.341 & 0.179 & 0.084 & 0.037 \\
$T_{p o t}^{\prime}$ & 2.87 & 0.974 & 0.597 & 0.420 & 0.248 \\
\hline \hline
\end{tabular}

$$
T_{C S E} \approx 2 T_{p o t}^{\prime}=\pi w / \sqrt{2 h_{p}},
$$

where the latter is the period of beam width oscillations for the quadratic potential approximation based on well depth $h_{p}$ but initial beam width $w$. As is to be expected, $T_{p o t}$ is of the right order of magnitude, but a consistent underestimate.

As seen in the next section, numerical results do show this spatial splitting with inner part having power just below $\mathcal{N}_{c r}$. So we observe that this condition for splitting and thereby collapse arrest is in qualitative agreement with the numerical results.

This mechanism for an initial inhibition of singularity formation does not rule out the possibility that self-focusing could bring the power of the inner part above the critical threshold on its slower time scale, after the initial inner focusing has produced one or more inner oscillations; this would lead to the pattern of one or more oscillations followed by singularity formation that is also observed above for potential just large enough to inhibit singularity formation in the initial beam collapse. Thus, to determine whether singularity formation can be permanently prevented, radiation needs to be considered, and a more detailed model of the radiation mechanism removing power from the region of the potential is needed.

\section{B. Radiation model for relaxation to subcritical inner bound states}

The collective coordinates method used for example to derive the form of the singular solutions of the CSE [17] and collapse inhibition by an attractive potential with moving off-center beam [6] can be used to study the evolution of beam width, describing the inner, focusing, part of the beam and its interaction with radiation to the outer, dispersing part. The approach is modified here to use a more accurate hyperbolic secant approximation of the ground state instead of the traditional Gaussian, for reasons explained below.

The related method of modulation analysis has been applied to other similar perturbation of the CSE, such as the addition of normal dispersion [18-20]. Its application in the current situation could be considered, but it would be a challenge beyound the scope of the present work, if it is possible at all. This is due to factors like the lack of spatial homogeneity and the resulting change in the solutions about which one perturbs. 


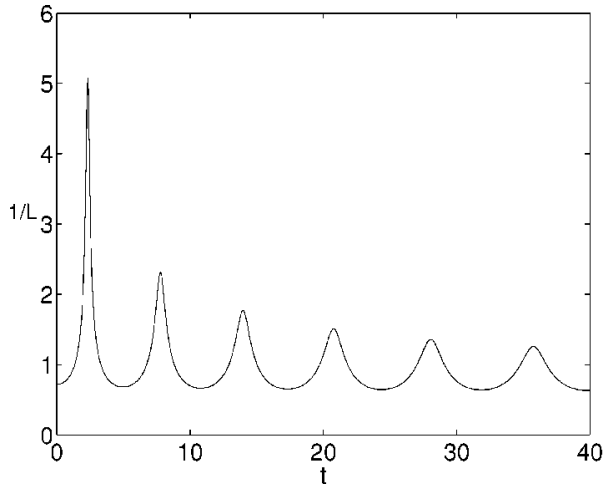

Omitting details, which are similar to these cited precedents, one describes the behavior in terms of a beam width scale variable $L$ and an excess of power over critical in the inner region, $\Delta$, related to the original equation through

$$
\begin{gathered}
\psi(t, \mathbf{x})=\frac{1}{L(t)} \Phi(\tau, \vec{\xi}) \exp \left(i \tau+i \frac{\dot{L}}{L(t)} \frac{r^{2}}{4}\right), \\
\vec{\xi}=\frac{\vec{x}}{L(t)}, \quad \frac{\partial \tau}{\partial t}=\frac{1}{L^{2}(t)}, \quad r=|\mathbf{x}|
\end{gathered}
$$

and

$$
\Delta=\frac{\mathcal{N}_{s}-\mathcal{N}_{c r}}{M} .
$$

Here, the width scale is determined by the condition that the transformed profile $\Phi$ is almost stationary and in the inner regions is approximated by the Townes soliton $R$, the stationary state profile for the CSE given by the positive radially symmetric solution of

$$
-\nabla^{2} R+R-R^{3}=0
$$

$\mathcal{N}_{s}$ represents the power within some inner region relative to the width scale,

$$
\mathcal{N}_{s}=\int_{|\mathbf{x}| \leqslant \xi_{s} L(t)}|\psi(t, \mathbf{x})|^{2} d \mathbf{x}=\int_{|\vec{\xi}| \leqslant \xi_{s}}|\Phi(\tau, \vec{\xi})|^{2} d \vec{\xi}
$$

$\mathcal{N}_{c r} \approx 11.67$ is the power of the Townes solition, the critical power needed for singularity formation, and

$$
M=\frac{1}{4} \int|\vec{\xi}|^{2} \Phi^{2} d \vec{\xi} \approx 3.4
$$

By considering the radiation rate for the core power we eventually get the equations

$$
\begin{gathered}
\ddot{L}=-\frac{\Delta}{L^{3}}-\frac{1}{2 M} \frac{\partial}{\partial L} \mathcal{V}(L), \\
\dot{\Delta}=-\frac{\gamma}{L^{2}}\left(\frac{N_{c}}{M}+\Delta\right),
\end{gathered}
$$

with the effective potential

$$
\mathcal{V}(L)=\int U(L \mathbf{x}) R^{2}(|\mathbf{x}|) d \mathbf{x},
$$

FIG. 2. Collective coordinates approximations with the sech approximation of the Townes soliton, $\quad L(0)=1.4, \quad L^{\prime}(0)=0, \quad \Delta(0)$ $=0.07, w_{p}=0.5$, and $h_{p}=5$. and $H(\beta)$ is the Heaviside function. These equations show that the width dynamics controls the tunneling rate and in this way modifies the core power kinetics.

If one now approximates $R(r)$ with a Gaussian of the form

$$
R(r) \approx R_{g}(r)=\sqrt{\frac{\mathcal{N}_{c r}}{\pi B^{2}}} \exp \left(-\frac{r^{2}}{2 B^{2}}\right), B^{2} \approx 0.8,
$$

one can evaluate the integral in Eq. (11), getting the closed form

$$
\frac{1}{M} \mathcal{V}(L)=-\frac{N_{c}}{M} \frac{h_{p} w_{p}^{2}}{2 w_{p}^{2}+B^{2} L^{2}}=-\frac{4.3 h_{p} w_{p}^{2}}{2.5 w_{p}^{2}+L^{2}} .
$$

However, as the resulting system can still only be studied numerically, it is better in this case to solve numerically using a more accurate model, at the cost of having numerical quadratures at each time step. Using a numerical approximation of $R(r)$ would be the most accurate, but it is observed that the intermediate approximation with a hyperbolic secant,

$$
R(r) \approx R_{s}(r)=K_{s} \operatorname{sech}(r / B),
$$

does not significantly reduce accuracy beyond what is already inherent in the ordinary differential equation (ODE) model. In comparisons, use of the hyperbolic secant consistently gives somewhat better fits to the NLSP than the Gaussian, so only this is used here.

We compared to the case $h=1.95, w_{p}=0.5$ where inhibition occurred for $h_{p}$ above 0.12 , and compared to that for $h_{p}=0.14$ as shown in Fig. 1 . The equivalent initial conditions for ODE's are

$$
L(0)=1.4, \dot{L}(0)=0, \Delta(0)=0.07,
$$

but collapse inhibition does not occur until about $h_{p}=2.3$, and to match more closely the partial differential equation (PDE) behavior for $h_{p}=0.14, h_{p}=5$ is used in the ODE. For comparison to the ODE amplitude data, $1 / L$ is plotted in Fig. 2 as well as the power surplus $\Delta$. This shows fairly good qualitative agreement with the full solution of the PDE, with the major discrepancy being an underestimation of the radiation rate, requiring the deeper potential. In particular the reduction of power in the inner region below the critical threshold is observed to be sharply the criterion for collapse 


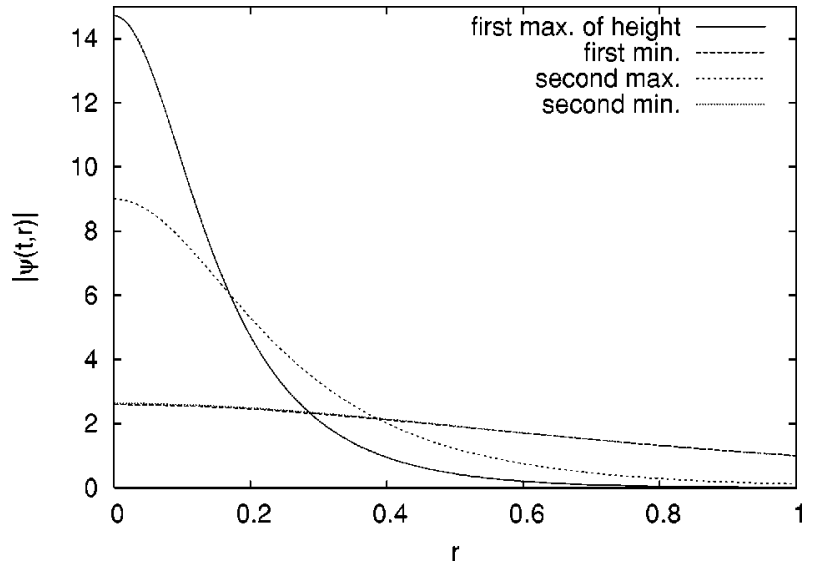

FIG. 3. $h=1.95, h_{p}=0.14$, and $w_{p}=0.5|\psi(t,)$.$| profiles showing$ oscillations in Townes soliton form.

inhibition in both the NLSP and the collective coordinates model.

\section{NUMERICAL RESULTS ON RADIATION FROM OSCILLATING BEAMS}

The main results here are a study of the observed transverse structure of solutions, which are seen to conform qualitatively to the above theoretical model.

Several observations can be made.

\section{A. Width oscillation in trapped beams with near ground-state form}

As shown in Fig. 3 for our "standard case" $h=1.95, h_{p}$ $=0.14$, and $w_{p}=0.5$ (and for other cases in [4,5]), when a potential prevents collapse or dispersal, the beam instead has oscillations in width and height, and width amplitude profiles following roughly a dilation pattern at small radii of the form

$$
|\psi(t, r)| \approx \frac{1}{L(t)} \widetilde{R}\left(\frac{r}{L}\right)
$$

for an asymptically oscillatory width scale $L(t)$ and spatial profile $\widetilde{R}$ somewhat close to the ground state $R$ but with power slightly below the critical value and, therefore, potentially close to stable bound states $u(r, L)$ of NLSP, at least when $L$ is small. This supports the approximation used for the inner region part of the collective coordinate model.

\section{B. Near null of intensity separating inner and outer parts}

At each minimum of beam width in an oscillatory solution, the intensity drops very close to zero at a "separation point" at approximately the potential's width, as shown for our standard case in Fig. 4, where the three curves at lower left are at the first three minima of beam width while the other two are at intervening width maxima. Figure 5 shows

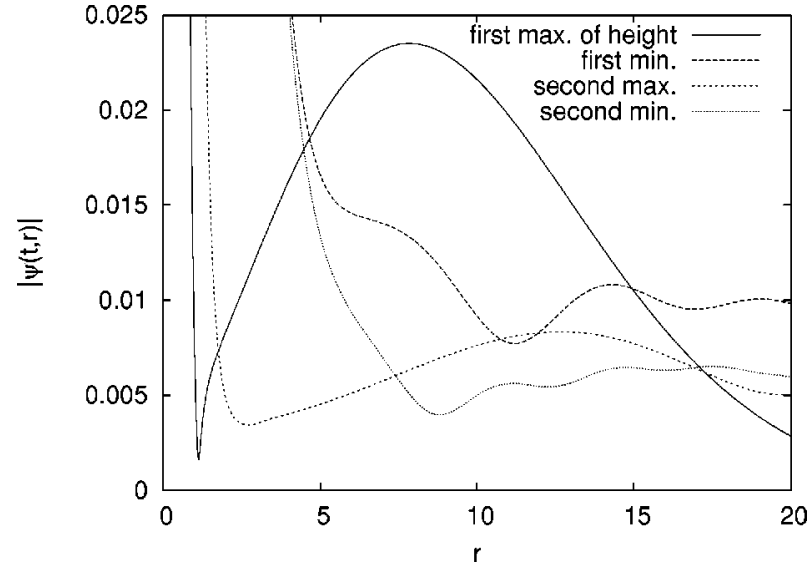

FIG. 4. $h=1.95, h_{p}=0.14$, and $w_{p}=0.5|\psi(t,)$.$| profiles: inner-$ outer separation.

the same for a beam with higher initial power, leading to considerably more power outside this separation point. In each case the intensity at the separation point is less than one thousandth of its maximum.

\section{Radiation}

Beyond the central oscillating spike and this separation point, one observes waves radiating outwards (see Fig. 6), carrying power well beyond the potential and spreading to such low intensities that nonlinearities are unimportant; thus the self-focusing effect will not refocus this power. Accordingly we find that the radiation is well described by the linear dispersion relation of the CSE, which at large radii reduces to $\omega=k^{2}$.

\section{CONCLUSIONS}

The previously observed inhibition of self focusing collapse by the addition of a small attractive Gaussian potential to the focusing cubic Schrödinger equation in critical

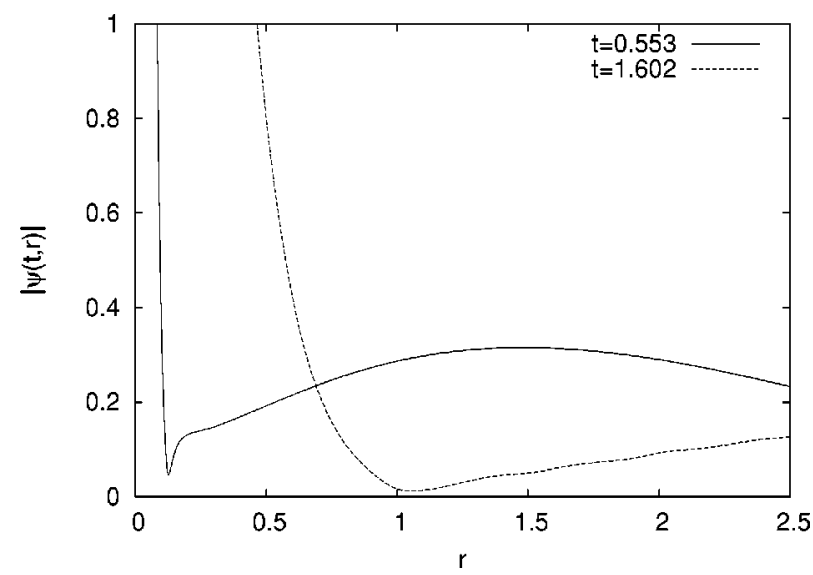

FIG. 5. $h=2.1, h_{p}=3.48$, and $w_{p}=0.3,|\psi(t,)$.$| with extreme$ splitting of beam power; near maximum $[|\psi(0.553,0)|=115]$ and minimum $[|\psi(1.602,0)|=11]$ of focusing oscillations. 

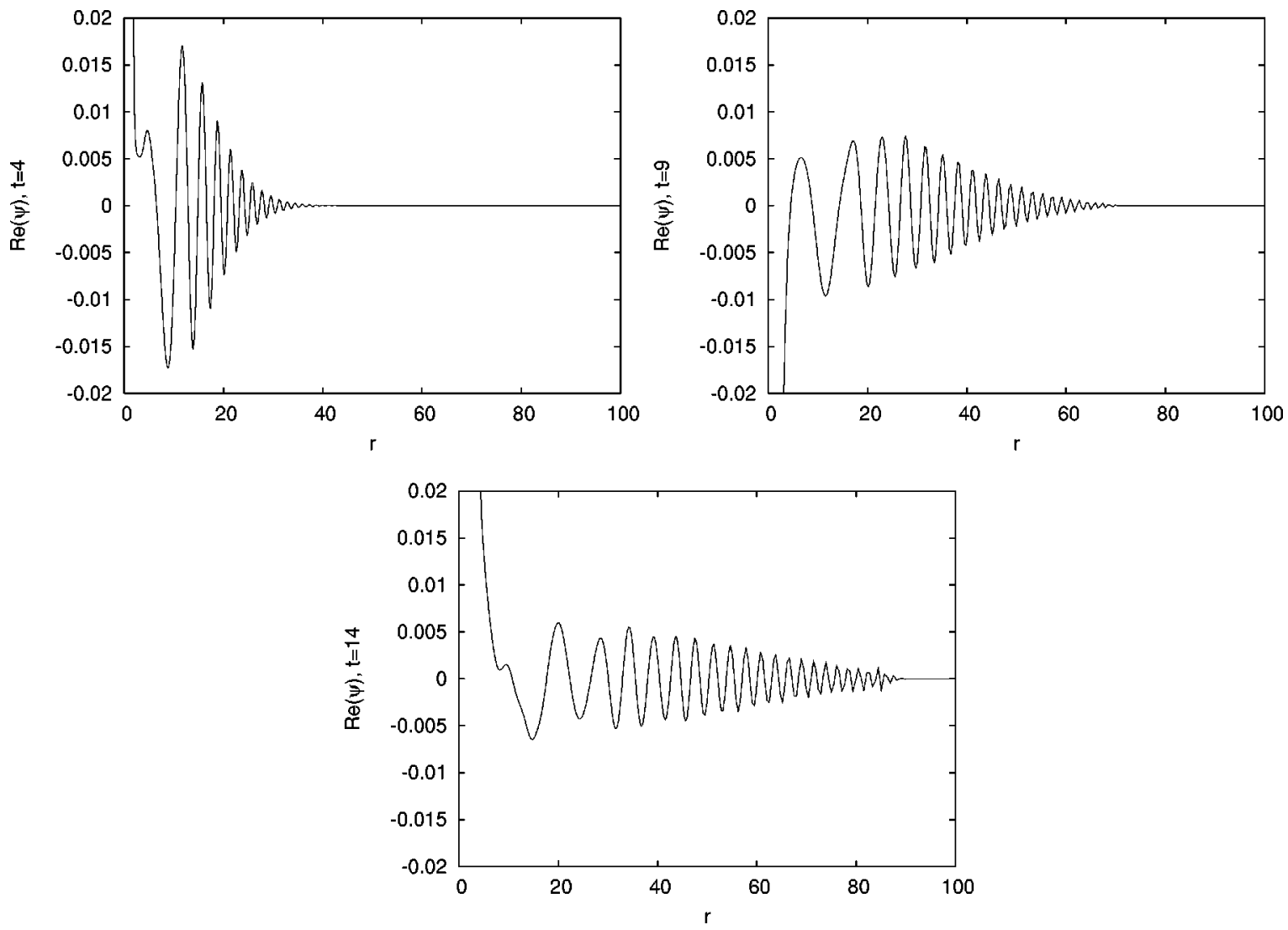

FIG. $6 . h=1.95, h_{p}=0.14$, and $w_{p}=0.5 \Re(\psi)$ vs $r$ at $t=4,9,14$, showing radiation.

dimension 2 has been qualitatively explained by the fact that linear focusing due to the potential occurs faster than the self-focusing collapse, leading to the amount of beam power entering the inner region where focusing occurs being less than the critical power needed for singular collapse, while the rest is left outside the potential well, at intensities too low for self-focusing to draw it into the inner region, so that it is instead dispersed radiatively.

Further from the potential, radiation is seen at each inward cycle of such oscillations, particularly the first.

This mechanism has been modeled via the lens transformation and a collective coordinate reduction, successfully predicting the main quantitative feature of beam width oscillations that decay somewhat, the power within the potential well becoming subcritical and hence preventing singularity formation, and power loss form the central focus through radiation at each oscillation.
Singularity inhibition is observed to occur with extremely narrow potentials, where the initial power outside the potential region is itself above critical, and so initial focusing involves a supercritical amount of beam power. This has been explained by a mechanism in which initially, self-focusing occurs with little affect from the potential, until a substantial proportion of the beam power has come into the potential region, after which the above pattern of splitting to a subcritical focusing core and a radiative outer part occurs.

\section{ACKNOWLEDGMENTS}

B.L. received financial support from the Danish Natural Research Council (Grant No. 21-02-0500) and a South Carolina Research Initiative Grant, and also thanks his hosts at the Danish Technical University. Yu.B.G. thanks Informatics and Mathematical Modelling, The Technical University of Denmark for a Guest Professorship.
[1] Y. B. Gaididei, K. Ø. Rasmussen, and P. L. Christiansen, Phys. Rev. E 52, 2951 (1995).

[2] L. Pitaevskii, Sov. Phys. JETP 13, 451 (1961).

[3] S. O. Demokritov, B. Hillebrands, and A. N. Slavin, Phys. Rep. 348, 441 (2001).

[4] B. J. LeMesurier and P. L. Christiansen, Physica D 184, 226 (2003).

[5] P. L. Christiansen, Y. B. Gaididei, and B. J. LeMesurier, in
Proceedings of "Localization and Energy Transfer in Nonlinear Systems,” edited by L. Vázquez, R. S. MacKay, and M. P. Zorzano (World Scientific, Singapore, 2003), pp. 28-43.

[6] Y. B. Gaididei, J. Schjødt-Eriksen, and P. L. Christiansen, Phys. Rev. E 60, 4877 (1999).

[7] J. Schjødt-Eriksen, Y. B. Gaididei, and P. L. Christiansen, Phys. Rev. E 64, 066614 (2001).

[8] B. J. LeMesurier, Math. Comput. Simul. 55, 503 (2001). 
[9] J. J. Rasmussen and K. Rypdal, Phys. Scr. 33, 481 (1986).

[10] J. J. Rasmussen and K. Rypdal, Phys. Scr. 33, 498 (1986).

[11] C. Sulem and P.-L. Sulem, The Nonlinear Schrödinger Equation: Self-focusing and Wave Collapse (Springer, Berlin, 1999).

[12] L. Bergé, Phys. Plasmas 4, 1227 (1997).

[13] L. Bergé, T. J. Alexander, and Y. S. Kivshar, Phys. Rev. A 62 , 023607 (2000).

[14] L. Pitaevskii, Phys. Lett. A 221, 14 (1996).

[15] T. Tsurumi and M. Wadati, J. Phys. Soc. Jpn. 68, 1531 (1999).
[16] H. Rose and M. Weinstein, Physica D 30, 207 (1988).

[17] M. J. Landman, G. C. Papanicolaou, C. Sulem, and P.-L. Sulem, Phys. Rev. A 38, 3837 (1988).

[18] G. Luther, A. Newell, and J. V. Moloney, Physica D 74, 59 (1994).

[19] G. Fibich, V. M. Malkin, and G. C. Papanicolaou, Phys. Rev. A 52, 4218 (1995).

[20] G. Fibich and G. C. Papanicolaou, SIAM (Soc. Ind. Appl. Math.) J. Appl. Math. 60, 183 (1999). 